\title{
A case of lipoatrophic panniculitis in a 2-year-old boy
}

\author{
Maria Weryńska-Kalemba ${ }^{1}$, Michał Kalemba ${ }^{2}$, Agata Filipowska-Grońska ${ }^{1}$, Anna Lorenc ${ }^{1}$, Jerzy Jarząb ${ }^{1}$, Andrzej Bożek ${ }^{1}$ \\ ${ }^{1}$ Chair and Clinical Department of Internal Diseases, Dermatology and Allergology in Zabrze, Medical University of Silesia in Katowice, \\ Poland \\ 2Department of Nuclear Medicine and Endocrine Oncology, MSC Memorial Cancer, Zabrze, Poland
}

Adv Dermatol Allergol 2016; XXXIII (2): 155-156

DOI: 10.5114/ada.2016.59165

Lipoatrophic panniculitis is generally a rare condition affecting mainly children, sometimes associated with connective tissue diseases [1, 2]. There are also reports about this condition occurring in adults [3]. This idiopathic, rarely reported panniculitis is presumed to have an autoimmune pathogenesis. A histopathological analysis reveals lobular panniculitis with fat necrosis and an associated inflammatory infiltration. Other histological findings are a focally increased number of lymphocytes rimming the adipocytes and infiltration of vascular walls by mildly atypical lymphoid cells [4]. Clinical and histopathological features lead to diverse diagnostic considerations like subcutaneous panniculitis-like T-cell lymphoma, Weber-Christian disease or Rothman-Makai syndrome [5].

We present a 2-year-old boy with no clinical or laboratory features of connective tissue disease who presented a progressive appearance of annular atrophic plaques with erythema on the right ankle. Skin lesions were about 2-3 cm in diameter with palpable loss of subcutaneous tissue (Figure 1). The patient was referred to the Chair and Clinical Department of Internal Diseases, Dermatology and Allergology in Zabrze after several consultations in other dermatology and rheumatology departments, with a suspicion of morphea, granuloma annulare or lipodystrophy. All blood tests like blood count, biochemical analysis, antinuclear antibodies and inflammatory markers were normal. Also X-ray of the lower limb was correct. There was no anamnesis of arthritis, thyroid gland abnormalities or fever. The first histopathological examination revealed acanthotic epidermis, collagen fiber protrusion, reduction of elastic fibers and in subcutaneous tissue - panniculitis. On the basis of the histological image, morphea profunda was suspected. However, the clinical picture with no immunological markers of connective tissue disease was still thought- provoking. The boy was treated with topical and systemic steroids for 6 months, and also with pentoxifylline and naproxen with no significant improvement. The second histopathological examination showed lymphocytes and histiocytes infiltration in the deep layers of dermis and subcutaneous tissue, in fatty tissue atrophy and inflammatory infiltration with histiocytes, lipofages similar to Touton cells and lymphocytes (Figure 2). On the basis of analyzed data, clinical and histopathological examination we identified lipoatrophic panniculitis. The appearance of new disease outbreaks required different treatment. Methotrexate (MTX) in an oral dose of $6.125 \mathrm{mg}$ once a week $\left(10 \mathrm{mg} / \mathrm{m}^{2} /\right.$ week) was administered for 10 weeks. A good tolerance of medication, on the basis of clinical condition and biochemical parameters monitoring, was noticed. We observed total suppression of the process with no further progression of skin lesions during 2 years of observation.

We present the case to describe clinical and diagnostic difficulties in our young patient with good clinical response to methotrexate (MTX) treatment. Although lipoatrophic panniculitis is a rare condition it should be considered particularly in the case of children. Panniculitis in children is a heterogeneous group of diseases, and so is in adults; it is characterized by inflammation of the subcutaneous and fatty tissue. Only a few types of panniculitis are found in childhood only, such as fat sclerosis of the newborn, while the vast majority of other types may be found both in the pediatric age and in adults [6, 7]. Lupus panniculitis, cold panniculitis, panniculitis in Behçet's disease, and post-steroid panniculitis are mainly observed in adults [8]. There are also few rare forms of panniculitis, such as eosinophilic panniculitis or subcutaneous panniculitis T-cell lymphoma, which often give diagnostic difficulties but also mainly occur in adults [9]. Lipoatrophic panniculitis is most frequently observed in

Address for correspondence: Maria Weryńska-Kalemba MD, PhD, Chair and Clinical Department of Internal Diseases, Dermatology and Allergology, Medical University of Silesia, 10 M. Skłodowskiej-Curie St, 41-800 Zabrze, Poland, phone: +48 664968021 , fax: +48 3227131 65, e-mail: maria.werynska@gmail.com Received: 9.03.2015, accepted: 11.05.2015. 


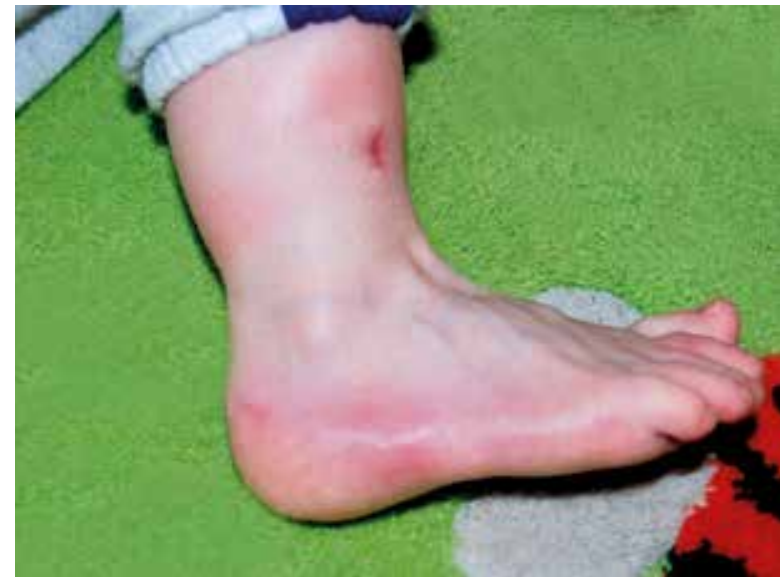

Figure 1. Skin lesions before the treatment

infants and children. Its clinical features are erythematous nodules and plaques followed by circumferential atrophy of the distal lower extremities, sometimes connected with fever, juvenile arthritis, or Hashimoto disease. In the diagnostic process it should be differentiated with all forms of panniculitis. Immunological, endocrine abnormalities and morphea or lupus profundus should be excluded. In our case, the patient was previously diagnosed with morphea, probably because of lack of experience in recognizing lipoatrophic panniculitis, which is rare. As described by some authors, it may be difficult to distinguish between lipoatrophic panniculitis and panniculitis of Weber-Christian or its variant - RothmannMakai panniculitis [10]. Histopathological examination of the deep skin biopsy seems to be crucial for differentiation. Also treatment of lipoatrophic panniculitis may be challenging. There are few reports concerning therapy with steroids, hydroxychloroquine or methotrexate (MTX) [11]. However, there are no clear indications for children's therapy.

Lipoatrophic panniculitis should be considered in infants and young children with clinical features of panniculitis and fat atrophy without clinical or serologic evidence of connective tissue disease. Methotrexate may be considered as a good treatment when systemic therapy is essential.

\section{Conflict of interest}

The authors declare no conflict of interest.

\section{References}

1. Grassi S, Borroni RG, Brazzelli V. Panniculitis in children. G Ital Dermatol Venereol 2013; 148: 371-85.

2. Ng SY, D'Arcy C, Orchard D. Acquired idiopathic lipoatrophic panniculitis in a 12-month-old infant. Australas I Dermatol 2015; 56: e102-4.

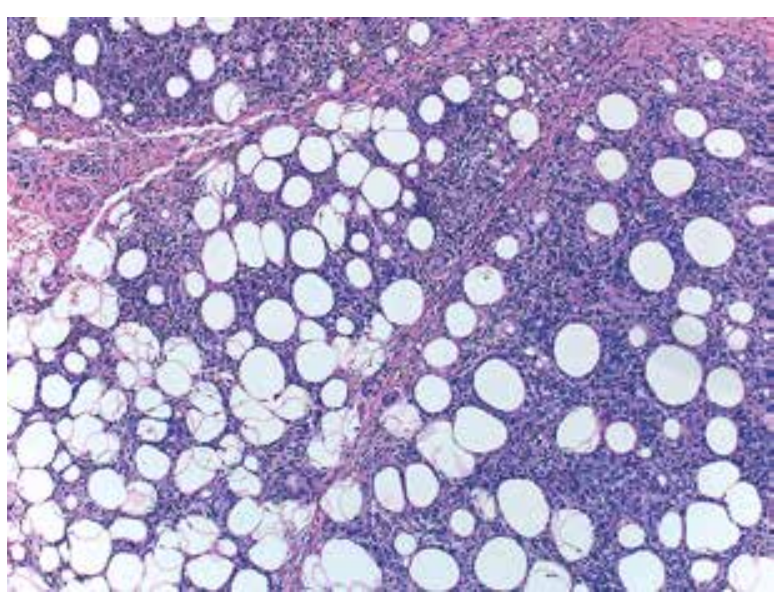

Figure 2. Histopathological examination - lipoatrophic panniculitis

3. Moulonguet I, Braun-Arduz P, Plantier F, et al. Lipoatrophic panniculitis in adults: treatment with hydroxychloroquine. Ann Dermatol Venereol 2011; 138: 681-5.

4. Dimson OG, Esterly NB. Annular lipoatrophy of the ankles. J Am Acad Dermatol 2006; 54: 40-2.

5. Labeille B. Panniculitis in children. Ann Pediatr 1992; 39: 419-25.

6. Handfield-Jones SE, Stephens CJ, Mayou BJ, et al. The clinical spectrum of lipoatrophic panniculitis encompasses connective tissue panniculitis. Br J Dermatol 1993; 129: 619-24.

7. Szczerkowska-Dobosz A, Olszewska B, Lemańska M, et al. Acquired facial lipoatrophy: pathogenesis and therapeutic options. Postep Derm Alergol 2015; 32: 127-33.

8. Bednarek A, Bartoszak L, Samborski W. Case report on a patient with lupus panniculitis. Postep Derm Alergol 2015; 32: 59-62.

9. Sokołowska-Wojdyło M, Olek-Hrab K, Ruckemann-Dziurdzińska K. Primary cutaneous lymphomas: diagnosis and treatment. Postep Derm Alergol 2015; 32: 368-83.

10. Santonja C, Gonzalo I, Feito M, et al. Lipoatrophic panniculitis of the ankles in childhood: differential diagnosis with subcutaneous panniculitis-like T-cell lymphoma. Am J Dermatopathol 2012; 34: 295-300.

11. Shen LY, Edmonson MB, Williams GP, et al. Lipoatrophic panniculitis: case report and review of the literature. Arch Dermatol 2010; 146: 877-81. 